day, $\frac{1}{2}$ hourly night, 8 to 10

Cystoscoped; ulceration of bladder.

T.B. found in urine. Patient weak, tired, and unflt for any work. Tuberculin treatment August 1st, 1919: 0.01 c.cm. P.T.O. to Jan. 13 th, $1920 ; 1 \% 3$ c.cnr. Old T. Marked genelal improvement in health. Able to do own housework. Much less pain. No hematuria. Better nights. Frequency, lay, 1 to $1 \frac{1}{2}$ hour's.

Mr. F. C., 30. May, 1919: Constant pain both kidneys and groin for a year ; not relieved by removal of two small stones from bladder per wethran six months ago. Now complains constant pain ancl weakness. Frequency, day, every 3 homs. Cystoscoped; T.B. around R.U.O. No T.B. found in mine. October, 1919: Slight reaction to 0.001 c.cm. Old T. Marked reaction three days later to $0.003 \mathrm{c.cm}$. Old 'T. Started treatment Nov. 1st, 1919: P.T.O. 0.0005 c.cm.; now reached $0.7 \mathrm{c.cm}$. P.T.O. Pain occasional only, and very slight. Frequency, $\frac{\text { day, } 3 \text { or } 4}{\text { night, } 0}$. Feels strong and well; able to work.

Mrs. A. W., 33. August, 1919: Tired, sleepless, and worm out. Practically no control over urine, passing; every 10 minutes day and night; great pain and smarting: frequent hematuria. Five year's ago right kidney was removed for tubercle; two sinuses have persisted since, and are now freely discharging. July, 1916 : Cystoscopic examination showed ulcerations of bladder wall : T.B. found in urine. Tuberculin treatment from August 1st to end of December, 1919 : P.T.O., P.T... and old T. to $1 \% 3$ c.cm. Wonderful improvenent, cheerful and energetic and happy; able to do her housework. Frequency, day. every 1 to $1 \frac{1}{2}$ hours

Pain occasional only. The sinuses are nearly healed. Very little discharge. Narch, 1920: Improvement maintaincd.

Mr. W. W., 38, husband of A. W., above. Ausust, 1919: Pain in left renal region tive years, increased by work. Frequency, $\frac{\text { day, every } \frac{1}{2} \text { hour }}{\text { night. 0-1 }}$. Hrinaturiaonce, copious, 18 months ago. Pain, chiefly after micturition, nearly constant. Cystoscoped ; no stone, no growth; ? tuberculous nodules. X ray, left kidney nil. T.B. found in urine. Tuberculin treatment, Anoust, 1919, to February, 1920: P.T.O., P.T., and Old T. to 1 c.cm. "Feels better than he has done for agses." Frequency, $\frac{\text { day. } 3-4}{\text { night, } 0}$. No pain; able to do full work.

Miss E. F., 35. A negative case. History: Five years" weakness of bladder. Micturition every half hour by day, once or twice only by night; no pain ; no hæmaturia. Tested with tuberculin up to $0.08 \mathrm{c} . \mathrm{cm}$. old T. (twice). No reaction whatever to any test. Clearly no tuberculosis.

Unsuitable cases for the treatment are found to be the following : 1 . Those in which any continued ferer be present; such fever is caused by mixed infection, and the patient should be kept in bed till the temperature is normal. 2. Albuminuria; no injections should be given. 3. Epilepsy; in such cases it is as well to decline treatment. Pregnancy is no bar to the treatment.

A common lesson to be drawn from these cases is as follows.

(1) Early cystoscopic examination along with testing by tuberculin should be practised in every suspicious case.

(2) Treatment of all cases, however long standing.

(3) Treatment can be carried out at dispensaries, a great economic gain to the community, patients usually being able to continue their work.

(4) The attendance of patients twice a week is essential for the proper progression of doses.
I am aware that medical men have tried the use of tuberculin in these and analogous cases, and have discontinued it, disappointed at the results. To my mind, the failure has been due in some of of these zases to the method in which tuberculin has been administered. I adopted the technique laid down by Dr. Camac Wilkinson, from whose teaching one of the first lessons which emerges is that neither patient nor physician should be afraid of reactions. The high temperature with unpleasant symptoms for 24 hours leads to no harm, and, in point of fact, is an invariable prelude to improvement. I must express my thanks for the assistance I have received from Dr. Camac Wilkinson, who placed his lengthy experience of tuberculin treatment at my disposal, and to Mr. Canny Ryall, through whose courtesy I was able to carry out the treatment of some 40 cases at All Saints Hospital.

(irafton-street, $\mathrm{W}$

\section{AN EXPERIMENT ON THE}

\section{M()DE OF TRINAMISLION OF CLRTAIN HLMAN INTENTINAL PROTOTOA.}

BY J. C. WATT, M.C.. M.B., CH.B. GLASG.,

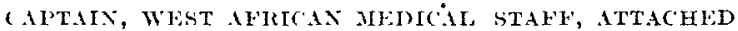

$$
\text { KINC'S AMMIT AN RIVLES. }
$$

The conclusions of Wenyon and O'Connor as a result of that part of their work in Egypt ${ }^{1^{\circ}}$ which dealt with the viability under various conditions of cysts of human intestinal protozoa, and of their mode of transmission from one host to another, appeared to negative my own, particularly on the mode of transmission of amcbic dysentery. For reasons subsequently to be stated my experience in West and East Africa led me to believe that amcobic infection was likely to be wind borne, and to lie in the dust of the countries where the disease is prevalent.

Wenyon and O'Connor, however, apparently showed that cysts of rarious intestinal protozoa, including that of Entamoba histolytica, are killed by drying or insolation; they showed that such cysts are frequently ingested by house-flies, and deposited with the freces of the latter wherever these may alight and feed; the main conclusion being that the ultimate channels of transmission of amobic dysentery are moist and wet food and water.

The test used by these observers to ascertain the viability of various cysts after drying was the eosin test. Cysts which stained with dilute eosin were considered to be dead, those refusing the stain to be alive. The reliability of this test seemed to me doubtful. It may be true that "a living cell will not stain with dilute eosin, whereas a dead cell will stain at once"; but does a cyst, which is not merely a cell, but also a cell provided with a resistant, although no doubt permeable outer layer or cover. ing, behave in the same way in this respect as will an unprotected tissue cell? It seems possible that a cyst, having lost fluid or having undergone a change in the fluid tension of its contents, but being still viable, may take up a nocuous diffusible substance, such as eosin, on being immersed in a solution of such a. substance. Any such interchange, however, of diffusible substances between the interior of a cyst and its surrounding medium must depend on the permeability of the cyst "wall."

1 Human Intestinal Protozoa in the Near Fast, by Temporary Lientenant-Colonel C. M. Wenyon, B.Sc., M.B., B.S., R.A.M.C., and 
Now, if it were possible to treat a cyst in such a way as to render its "wall" an impermeable membrane, without damage to its living contents, such a cyst would resist staining. In this connexion one of the findings of Wenyon and $O$ 'Connor is significant. They found that exposure of histolytica cysts to various chemical solutions caused them to stain with eosin; but that exposure to 1-100 formalin failed to cause the cysts to stain, "even after four hours ...... though they were very much shrunken and distorted, and gave every appearance of having been killed." It is likely that the resistance to staining in this case was produced by the hardening effect of the formalin on the cyst wall, rendering the latter impermeable, and so precluding the stain from access to the cyst contents.

If this is the true explanation of the phenomenon it seems possible that in conditions resulting in the gradual hardening and drying of such cyst walls-for example, insolation and desiccationthe cyst wall may succeed in preventing damage to the contents of the cyst; in other words, that such cysts may be viable, even although they may present the appearance, from wrinkling of the envelope, of having been killed. This seems the more probable when we consider that the cysts of free-living but closely related creatures remain viable under desiccation. As Wenyon and O'Connor point out, "the Thompsons have shown that the free-living Amaba limax produces a cyst which will withstand complete and prolonged desiccation under a tropical sun.'

\section{Description of Experiments.}

In the experiment I have recently carried out the materials used first were :-

1. Fluid fæces-produced after purgation with mag. sulph.-in which the following organisms were seen :-

(a) Tetramitus (Chilomastix) mesnili-numerous.

(b) Trichomonas intestinalis-not so numerous as the former.

(c) A small amoba, sluggishly motile, often extruding several blunt pseudopodia at once, showing a granular endoplasm, the pseudopodia being ectoplasmic, of low refractibility; size equal to, or slightly less than, that of an R.B.C. These I thought might be the $E$. nana of Wenyon and O'Connor, but could not prove this by stained specimens.

(d) Cysts, presumed to be of the forms mentioned above, namely: 1. Clear, bottle-shaped, with one o two dark specks, which I diagnosed as $T$. mesnili. 2. Larger, clear, bluntly oval cysts, which I considered might be those of $T$. intestinalis. These mostly exhibited flattening of both sides and ends, and were not unlike lamblia crsts, though smaller and without visible internal structure. 3. Small, spherical, in size about equal to an R.B.C., with coarsely speckled contents, no other internal detail being visible.

2. Stephens's scarlet ink.

3. Rain water.

4. Double strength physiological saline solution.

5. 2 per cent. HCl solution.

The fæcal material was first exposed to the sun for one day, dried in the shade for three succeeding days.

Test A.-Small quantities were then emulsified on slides with rain water, and then, after the lapse of $5,10,20$, or 30 minutes' interval, wer'e mixed with an equal volume of scarlet ink.

Result.-Most cysts of all three types were seen to be stained.

Test B.-Again replacing the rain water by donble physiological saline, the same intervals being allowed before mixing with stain.

Result.-Most cysts of all types stained.
Te'st C.-(1) The same fiecal material (four days old not having been exposed to tho sun, but after drying on a cover-slip for four hours, was thon mixed first with (2) double physiological saline, and immediately also with (3) culture of paramocium, mostly living and moving actively, (4) scarlet ink.

Result.-One typical tetramitus cyst seen entirely unstained, all other material on the slide-including the paramoecia-being deeply stained, except some motile bacteria.

The reason for making these tests was as follows I considered the staining of dried but possibly still viable cysts might depend either on (1) their loss of fluid, or (2) their relatively high concentration of diffusible substances. Subsequent experiments, on the same lines, with similar materials, gave equivocal results, most of the cysts staining irrespective of what solutions they were treated with before being mixed with the staining solution. That is to say, having once been dried, even short of the point of complete desiccation, they could not be satisfied with an innocuous solution to the exclusion of the nocuous stain.

The same tests were gone through with lamblia cysts, but these all stained readily and completely after short periods of drying.

One result, however, of these tests was that a few cysts of the type described as those of tetra. mitus, which had been dried for anything between four and ten days, refused to stain, appearing perfectly colourless.

The original specimen of fæces was then placed aside and re-examined between one month and six weeks later, when a scale of dry powder remained in the flask, which had originally contained between 3 and 4 ounces (fi.) of fæcal material. Emulsified and examined under the oil immersion lens numerous cysts were seen on each slide observed. But although those cysts diagnosed as of $T$. mesnili had been fairly easily found in the fresh material, these were now apparently absent, all cysts seen being curiously alike, and spherical or subspherical, except that a few of the larger oval cysts originally present were still to be found. The majority of cysts seen, however, were about equal in size to an R.B.C.; none showed the lobe or knob charac. teristic of those of tetramitus. Further, all cysts appeared somewhat crenated, and had lost the glassy appearance they had had when fresh.

Some, however, appeared as shown in Figs. 1 and 2. They appeared as if they might have
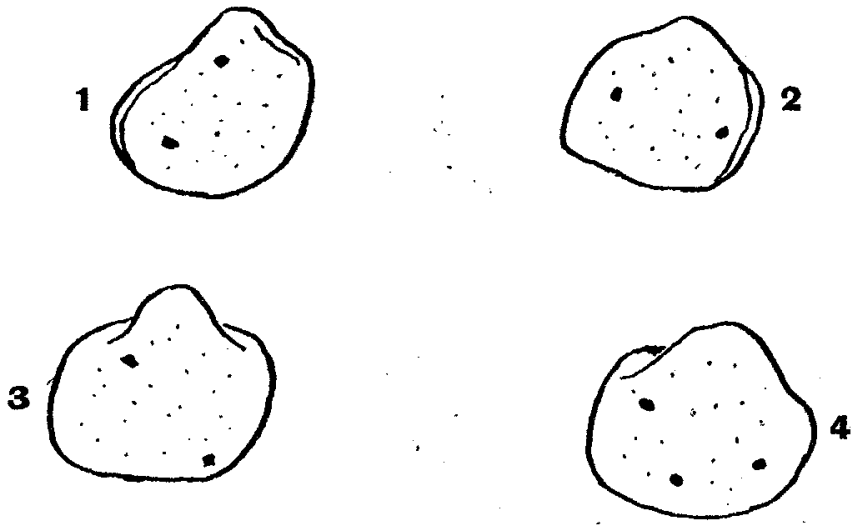

originally possessed a distinct lobe which had been retracted during desiccation into the main part of the cyst. Indeed, a few gave the appearance shown in Figs. 3 and 4. Later, after immersion in 0.2 per cent. $\mathrm{HCl}$, some of these cysts actually seemed to "fill out," as if resuming the form showing a distinct knob or protuberance. 
Test D.-A small quantity of this ariol matcrial being now emulsified with sterile water and mixed with an equal volume of scarlet ink was oxaminert muder the microscope with surprising results. The largo majority of the cysts were entively unstained. In the repeated examination of many such specimons a cyst was occasionally seen which appeared to be slighitly tinged with the stain, but not one was found deeply stained. All the other's appored perfectly clear and colourless against the red or pink background of the slide. After four days vaselined specimons showod tho cysts to be still unstained.

Test E.-The same emulsion heated on a slicle over a spirit lamp and then stained gave the following result: (1) within half an hour, all cysts slightly tinged; (2) after 24 hours, all cysts stained a deep redilish brown.

Test $F$.-Some of the frcal material was emulsified in plain sterile water in small flasks and examinod after 24 hours. It was then seen that a large majority of all the cysts had leveloped into motile amoebula, which were, to inspection at least, similar to those I had observed in the fresh freces; but I concluded that these must be free-living amœba, which had either been present in the original fresh stool, or were morely a contamination, having been carricd into the frecal material during the process of drying. I was now doubtful as to the survival of any true tetramitus or trichomonas cysts, but proceeded to a fecding test.

Test G.-A chicken, about 1 month old, apparently healthy, was kept in a cage for five days, its droppings examined while newly passed, both unstained and stained with an equal mixture of 5 per cent. iodine in pot: jod. and Stephens's scarlet ink. No protozoal or other organisms were seen, except bacteria. The chick was then made to 'swallow, morning and evening, about gr. i.-ii. of the dried freces originally used-that is, material about two months old. Twenty-four hours after the commencement of the test the chick developed diarrhoea, with mucus in the droppings, but no cellular exudate. The following organisms were then found in the mucus: (a) Typical motile trichomonas, numerous. (b) Tetramitus, less numerous than the former, moving sluggishly. (c) One active amceba, about $6-8 \mu$ in diameter, moving sluggishly, with granular endoplasm, and not very sharply defined ectoplasm : refractility comparatively low. On the second day of infection, besides trichomonas, a few typically motile tetramitus were seen. On the third clay of infection the chick still showed diarrhoea in the morning, and the droppings contained large numbers-one or two to each field with a 1/12-inch objective-of tetramitus and trichomonas. Feeding with the freal material was stopped, the last dose being given at $6 \mathrm{~A}$.M. on the third day after that on which feeding was commenced. On the evening of the same day, diarrhoea had passed off, and only one or two motionless forms of tetramitus and trichomonas were found. Cysts of the types seen in the original fresh specimen appeared in fair numbers. On the fourth day the chick's droppings were again examined. No protozoa were observed, nor their cysts. The chick was then killed, and the contents of all parts of the intestinal canal examined microscopically. No protozoa were found, but in the material taken from the crop were several of the spherical and subspherical cysts as seen in the dried freces fed to the chick.

Two other experiments were carried out on chicks. In one, dried fæcal material, which had, when fresh, shown a fair number of tetramitus and trichomonas, with appropriate (?) cysts, was used. 'This material, - after about 18 days" drying, with occasional insolation (Lat. $2^{\circ}-3^{\circ}$ N.), but during very rainy weather, showed all cysts stained when treated with scarlet ink. No protozoal infection was induced in the chick. In the second material, sun-dried, containing $E$. histolytica cysts, failed to infect the chicken, even after 5-6 days' trial. In each case blood and mucus, with a heavy cellular exudate, appeared in the droppings of the chick a few hours after the commencement of feeding with the fæcal material, and the chick in both cases was definitely ill. Immediately, however, on cessation of feeding with the fæcal matter the dysenteric condition cleared up, and the chick entirely recovered.

\section{Conclusions.}

Since making these experiments I have had no opportunity of making confirmatory tests; but I feel that, in the case of the first chicken test I have described, there can be little doubt that the chicken was infected by the sun-dried material fed to it, for these reasons: (1) The exact correspondence between the organisms observed in the material fed to the chick and those appearing in the droppings of the chick ; (2) the correspondence between the duration of feeding with the test material and the duration of infection; (3) the absence of any intestinal protozoal infection from several other chickens examined, at intervals, over a period of three months, some of these chickens feeding in the hospital compound.

It is possible that the "positive" chicken may have ingested house-flies carrying protozoa or their cysts during the period of the test; but this was not observed and the chick was confined in a cage during the test. No cysts were to be found in the water given to the chick; it was fed with rice. It does not seem possible that the chick could have been so heavily and suddenly infected by the ingestion of, at most, one or two flies.

With regard to the dysentery induced in these chickens I conclude that it was probably entirely due to the irritation of poisonous substances in the fæcal material ingested.

Since making the staining tests described I have noticed that living organisms, such as tetramitus and trichomonas, which entirely refuse to be stained by scarlet ink when they are newly passed in the stool, will, while still showing motion, stain slightly with the same material after having been exposed to room temperature (between $70-80^{\circ} \mathrm{F}$. in this case) for a few hours, but without drying other than that due to ordinary eraporation of the fluid fæces containing them. It would appear, therefore, that staining of living organisms-cells-such as these is merely proportionate to the degree of loss of vitality.

I have ventured to make the following suggestions from these tests: (1) That the cysts of Trichomonas intestinalis and of Tetramitus mesnili may remain viable and capable of reproducing active forms after insolation under a tropical sun. (2) That the staining of certain intestinal protozoal cysts is inversely proportionate to the degree of drying and hardening of the cyst wall. (3) That staining cannot be accepted as a test of the viability of these cysts. (4) That the cyst of Trichomonas intestinalis is of a flattened oval shape (flattened as to both sides and ends), slightly larger than the cysts of T. mesnili, of clear, faintly spotted appearance when fresh, and exhibiting no internal structure when fresh. I regret I have not been able to use the appropriate stains for these cysts.

It seems likely, therefore, that the infections of $T$. intestinalis and $T$. mesnili may be dust-borne, and I think the experiment I have described is sufficient to warrant a re-examination of the more serious question of the mode of transmission of amcbic dysentery. I have referred to my belief that this disease is dust-borne, and it will be admitted that there are many reasons for supposing this to be the truth. "Without going into detail, it is generally remarked that amobic dysentery is most prevalent in the driest and most dusty countries, and that it is most prevalent in the dry seasons of those countries. Further, I suggest that the onset of rains will cut short prevalent amcbic dysentery. I should like to refer 
to two presumptive instances of this. First, in the campaign in German East Africa the onset of the rainy season coincided exactly with a very great reduction in the numbers of cases of dysentery amongst the white troops with whom I was serving and of all cases amongst these $I$ found a very large proportion to be amoebic.

Again, in Gallipoli, it was remarked that the onset of the rain in August seemed to put a stop to the dysentery that had been so prevalent amongst the troops there, and although it has since been questioned that much of the dysentery during the Gallipoli campaign was amœbic, it is at least admitted that that peninsula must $j e$ regarded as an endemic centre of amobiasis. No one familiar with hot dusty countries-and especially with cam. paigning in these countries-can forget the welcome freedom from dust accompanying the onset of the rains. My inference is that the almost absolute freedom from dust in the rainy season suggests that dust has been the medium of infection where dysentery also ceases with the onset of the rains. It may be argued that the rains also reduce the numbers of flies, but a few hours' sunshine is generally sufficient to cause these to reappear and infest food materials as before.

Note.-On the question of the duration of infections of $T$. intestinalis and $T$. mesnili, one case I know to have lasted for over two years, including a period of four months in England. Cyst formation seems to be irregularly periodic in occurrence.

\section{Climital Alotes:}

MEDICAL, SURGICAL, OBSTETRICAL, AND THERAPEUTICAL.

\section{INTENSIVE IODINE TREATMENT.}

BY ToM Bird, M.A., M.R.C.S.

CONSUlTING ANESTHETIST TO GUY'S HOSPITAL.

Dr. Louis Bourdreau published in $1914^{1}$ his experience, of some 14 years' duration, of the intensive treatment of tuberculosis by large and continuous doses of the tincture of iodine of the French Codex. The article is remarkable for three things : his great success as to results in tubercu. losis, the large doses that can be given by a gradual increase, and the proof of sincerity in that Dr. Bourdreau did not publish his results until some 14 years after he began the treatment. His colleagues only had knowledge of his work, and he shuwed rare restraint in delaying publication for so long. He suggests the "intensive treatment of iodine" might be useful in other diseases. The article came under my notice through a letter of Dr. William Ewart, of St. George's Hospital, who resorted to the treatment for his own arthritis with such success that "from being unable to go upstairs one at a time, he could manage three at a time." $\mathrm{He}$ took $10 \mathrm{gr}$. in the day of pure iodine, through the medium of the French tincture (or Indian tincture), neither of which contain any iodide of potassium.

In quite a number of arthritic cases, and in three or four cases of chronic gout, and in those cases called "rheumatic gout," I have had a quite remarkable success, especially as to pain and insomnia. In $1917 \mathrm{I}$ met an old friend, Dr. J. H.
Ewart, of Eastbourne, and no relation of the above. mentioned Dr. Ewart, who told me he used the treatment, and "was continually having prescriptions returned by the chemists. who were surprised at the doses." Although he is himself susceptible to iodine, he told me that he could take $3 \mathrm{i}$ of the French tincture for a dose. He wrote :-

"I tried the treatment. tinct. iodine (French), and began with 10 min. and went on to 30 min. three times a day, and the results were really marvellous; my finger-joints decreased in size in a most wonderfu manner. I then went womg, and began taking $60 \mathrm{~min}$. three times a day, and this conced by giving me a diarrhoea I shall not forget. But to refer to other people. I treated at the All Saints Convalescont Hospital everrbody who complained of chromc rheumatism with tinct. iod. (F.). I don't think the three weeks they were there that I got boyond 30 min. ter die; but the results were really splendid."

With regard to tuberculosis, I hare only one case to report. He had been at a sanatorium, and was apparently cured; but in a comparatively short time began to fail, and at last came hæmorrhage. I had olready advised him to try iodine as a prophy. lactic. When the hæmorrhage came he was alarmed and began to take iodine. Some two months after I met him by accident and was surprised at his healthy appearance; he was, he said, quite well and had increased $10 \mathrm{lb}$. in weight. One swallow does not make a summer, but a swallow is a happy omen when it comes.

Some people are susceptible to iodine, as is my friend Dr. Ewart, of Eastbourne; yet he arrived at 3i. doses. I generally order $1 \mathrm{gr}$. after meals three times a day the first week, $2 \mathrm{gr}$. the second week, $3 \mathrm{gr}$. the third week. For arthritis I seldom give more. Beyond slight catarrh of the nasal mucous membrane, of the possibility of which I warn patients, I hear of no inconvenience, certainly no iodism. If the treatment has been left off it is better to begin again with small doses, as diarrhœa may follow a large dose. When pain and swelling have been relieved my custom is gradually to discontinue the medicine. It is un. fortunate that the article in the Journal de Médecine is out of print, and apparently unobtainable, as some of the details recorded are interesting and illuminating.

The notable absence of iodism following the use of the French tincture suggests that it might be used instead of iodide of potassium.

Conduit-street, W.

\section{A CASE OF OSTEITIS FIBROSA.}

By Norman C. Lake, M.D. Lond., F.R.C.S. ENG., STIRGYON, QUHEN'S YOSPITAL FOR CHILDREN, HACEYEY-ROAD, E; AND

Norah H. Schuster, M.B. Vict., HOL'SE SCHGLON, QLEEN'S HOSPITAL FOR CHILDREN.

THE chief point of interest in this case was that, although the $\mathrm{X}$ ray photograph so closely resembled that of Mr. E. G. Slesinger's case which appeared in, THE LANCET of Nov. 15th, 1919, and very strongly suggested cyst formation, both the operation and subsequent microscopy showed the complete absence of cysts.

The patient, a girl ased 7 years, attended the Queen's Hospital for Children in March, 1916, with a history of a fall three years previously and subsequent limping. There was no shortening of the limb or tenclerness, and a bony swelling was felt over the upper end of the femur. The $\mathrm{X}$ ray showed a coxa vara with questionablesigns of old tuberculons disease or periostitis. 